\title{
Análisis morfométrico del frenillo labial superior durante el crecimiento y desarrollo del niño en edades 0 a 12 años
}

Osmary Darisay Cerrato López;

Mónica Cristancho²;

Jonathan Liria

\section{Resumen}

Objetivo: Establecer un análisis morfométrico del frenillo labial superior durante el crecimiento y desarrollo del niño en edades de 0 a 12 años en el Municipio Naguanagua periodo Marzo-Septiembre 2016. Materiales y Métodos: investigación de tipo descriptivo con un diseño de campo. Teniendo como objeto una población de 650 niños tomando como Muestra 260 sujetos, distribuidos de manera porcentual en, 31\% Primera Infancia 23\% Segunda Infancia y 46\% Tercera Infancia. Se obtuvieron fotografías del frenillo, tomando 10 "landmark" (puntos de referencia) para la conformación de la estructura, siendo procesadas para su análisis con los Software, TpsDig2, Past, IMP7 y Morphoj. Resultados: la zona que presento mayor superposición en todos los grupos fue la de inserción gingival, mientras que la inserción labial presento mayor variación, en cuanto a la deformación de la estructura en todas las etapas hubo desarrollo en sentido horizontal en la inserción labial. La medida promedio del frenillo labial superior se estableció entre 11 a $13 \mathrm{~mm}, \pm 1-2 \mathrm{~mm}$ de desviación. La inserción gingival a la papila interincisal se presentó una medida promedio 1 a $3 \mathrm{~mm}$. $\pm 1 \mathrm{~mm}$. Conclusiones: el frenillo labial superior al nacer es fisiológicamente tectolabial, presentara un desarrollo de 1 a $2 \mathrm{~mm}$ durante el 1er año de vida en su zona de inserción gingival exclusivamente, manteniéndose estable sin reposicionarse ni modificarse en longitud hasta la adolescencia, por lo que al realizar el análisis Morfométrico del frenillo labial superior en todos tipos de dentición dicha estructura presento similitudes indicándonos su estabilidad en las diferentes edades del infante..

Palabras clave: frenillo, morfometría, crecimiento, desarrollo.

Artigo Original

\section{Análise morfométrica do freio labial superior durante o crescimento e desenvolvimento crianças em idades de 0 a 12 anos}

\section{Resumo}

Objetivo: Estabelecer uma análise morfométrica do freio labial superior durante o crescimento e desenvolvimento de crianças de idades 0 a 12 anos no município Naguanagua período Marzo-Septiembre 2016. Materiais e Métodos: Pesquisa de tipo descritivo com um projeto de campo. Tendo como objeto uma população de 650 crianças e tomando como amostra 260 sujeitos, distribuídos de

${ }^{1}$ Residente del Postgrado de Odontopediatría, Universidad de Carabobo.

${ }^{2}$ Odontopediatra UC. Docente del Postgrado de Odontopediatría U.C. y docente de Pregrado facultad de Odontología U.C.

${ }^{3} \mathrm{PhD}$, en Entomología, Docente de la Universidad de Ikiam Ecuador. 
forma percentual em, 31\% Primeira Infância 23\% Segunda Infância e 46\% Terceira Infância. Se obtiveram as fotografias do freio, tomando 10 pontos de referência para a conformação da estrutura, as quais foram processadas para seu análise com os software, TpsDig2, Past, IMP7 e Morphoj.

Resultados: a zona que preside o superposição do maior em todos os grupos foi a inserção gingival, enquanto que a inserção labial apresentou a maiori variação, forma da deformação da estrutura em todas as etapas desenvolvimento ocorreu no sentido horizontal na inserção labial. A medida médioa freio labial superior foi 11 a $13 \mathrm{~mm}, \pm$ $1-2 \mathrm{~mm}$ de desvio. A inserção gengival à la papila interincisal se mostrou uma medida média 1 a 3 $\mathrm{mm} . \pm 1 \mathrm{~mm}$. Conclusões: o freio labial superior , ao nascer, é fisiologicamente teto-labial, apresenta um desenvolvimento de 1 a $2 \mathrm{~mm}$ durante o primeiro ano de vida em sua zona de inserção gengival exclusivamente, mantendo-se estável sem reposicionar nem modificando em comprimento até a adolescência. Por isso que realizar analise morfométrica do frenillo labial superior em todos os tipos de dentição nos mostra semelhanças nos indicando sua estabilidade nas diferentes idades da criança.

Palavras Chave: freio, morfometria, crescimento, desenvolvimento.

\section{Original article}

\section{Morphometric analysis of the upper lip frenulum during growth and development of the child aged 0 to 12 years}

\section{Abstract}

Objective: To establish a morphometric analysis of the upper lip frenulum during the growth and development of the child aged 0 to 12 years in the Naguanagua Municipality period March-September 2016. Materials and Methods: A descriptive research with a field design was performed. The target population was 650 children, the sample taken was 260 subjects, distributed in 31\% First Infancy 23\% Second Infancy and 46\% Third Infancy. Photographs of the bridle were taken, taking 10 landmarks for the structure conformation, being processed for analysis with the Software, TpsDig2, Past, IMP7 and Morphoj. Results: the area that presented greater overlap in all groups was that of gingival insertion, while the labial insertion presented greater variation, in terms of the deformation of the structure in all the stages there was development in horizontal sense in the labial insertion. The mean upper lip frenulum measurement was established between 11 to $13 \mathrm{~mm}, \pm 1-2 \mathrm{~mm}$ of deviation. The gingival insertion to the interincisal papilla presented an average measure of 1 to $3 \mathrm{~mm}$. $\pm 1 \mathrm{~mm}$. Conclusions: the upper labial frenulum at birth is physiologicallyw tectolabial, presenting a development of 1 to $2 \mathrm{~mm}$ during the first year of life in its area of gingival insertion exclusively, remaining stable without repositioning or modification in length until adolescence, The morphometric analysis of the upper lip frenulum in all types of dentition said structure showed similarities indicating its stability in the different ages of the infant.

Key Words: frenillo, morphometry, growth, development 


\section{Introducción}

El frenillo labial superior es un pliegue sagital de la mucosa alveolar, en forma de una hoja de cuchillo, insertados por un lado en la superficie interna del labio y, por otro lado, en la encía, en la línea media de los maxilares entre los incisivos centrales. ${ }^{1}$ el mismo, según su forma, tamaño y posición está sujeto a ciertas variaciones determinadas por la estructura máxilofacial. Algunos pueden ser estructuralmente amplios y resistentes, otros son finos y frágiles.

Así mismo, el Frenillo Labial Superior (FLS) forma parte de una de las estructuras anatómicas de la cavidad bucal del niño, que de presentar alteraciones en su crecimiento y desarrollo, pueden ocasionar problemas como la dificultad al amamantarse, incompetencia labial, llegando a ocasionar una sonrisa gingival por labio corto, labio hipomóvil, dificultad en algunos fonemas en el habla, recesión de tablas óseas, y presencia de diastemas que pudieran retener la erupción de sus dientes vecinos por la amplitud del espacio interincisa ${ }^{1,2}$ siendo este último uno de los principales motivos para las intervenciones quirúrgicas del FLS, reflejado así en diferentes investigaciones de índole internacional donde se evidencia que 10 de cada 20 niños con presencia de diastema interincisal son sometidos a procedimientos quirúrgicos innecesarios, ya que diversos investigadores, establecieron que el cierre de diastemas se puede producir fisiológicamente en un 83,2\% de los casos, pudiendo dentro de esta cifra ocurrir diagnósticos erróneos que lleven a decisiones irreversible o que no aporten la solución real al problema de base. ${ }^{3}$ lamentablemente a nivel nacional no contamos con estadísticas que nos puedan orientar sobre la buena praxis o los resultados de los tratamientos relacionados al frenillo labial superior, sin embargo en la clínica diaria se hace evidente las dudas del odontólogo tratante o especialista al momento de la decisión quirúrgica.

De tal forma que, el estudio de los frenillos labiales y las consecuencias que pueden ocurrir a mediano y largo plazo evidencian las bases para determinar las dificultades que eventualmente se presentan en la detección temprana y por ende la intervención oportuna de los frenillos labiales en los infantes. ${ }^{4}$ La variabilidad de dicha estructura anatómica presentada bien sea por la edad, por el tipo de dentición o de factores asociados que influye en su morfología, suele ocasionar inconvenientes a la hora de un diagnóstico certero, la estandarización de ideal y lo patológico no termina siendo concluyente para la toma de decisión a la hora del tratamiento por lo que, El frenillo labial superior no cuenta más que con un ojo clínico que debe agudizarse a través de los años de experiencia, incluso a través del ensayo y error del especialista, para diagnosticar su forma, patología y su momento quirúrgico. ${ }^{5}$

Adicionalmente, la remoción del frenillo labial superior en el momento inadecuado puede desencadenar una fibrosis muscular de la zona que requiera a la larga un nuevo momento quirúrgico, así como no tener mejoras en lo que se consideraba patológico, haciéndose esencial para el diagnóstico establecer características cuantitativas y cualitativas fisiológicas a considerar en el crecimiento y desarrollo del individuo que nos ayuden a predecir si la evolución se está dando de manera normal o no y así hacer la intervención oportuna, y poder evaluar las distintas posibilidades de tratamiento. ${ }^{6}$

Los cambios morfológicos de las estructuras anatómicas en un individuo como el infante, suelen ser progresivos y lentos, a lo largo de los 
años pudiendo modificarse y adaptarse según los estímulos a los que es sometido durante todas sus etapas, siendo a veces poco perceptible sus variaciones y desviaciones durante su crecimiento y desarrollo, pudiéndose dificultar su seguimiento con el pasar del tiempo. De tal forma, el monitoreo durante su evolución toma importancia, a fin de establecer opciones terapéuticas para interceptar cualquier anomalía. ${ }^{7}$

Es por ello que el área de salud se ha visto en la necesidad de buscar herramientas que le permitan visualizar detalladamente los cambios del cuerpo humano, entre ellas se cuenta con la morfometría la cual se encarga de la covariación de la forma con factores subyacentes. Su desarrollo en las últimas décadas ha alcanzado áreas de la biología tradicionalmente dedicadas al estudio descriptivo, como las ciencias morfológica las que con los nuevos avances como la morfometría geométrica ha logrado no sólo objetivar la evaluación cuantitativa de los cambios morfológicos sino también la evaluación cualitativa a través de la recuperación de la forma en estudio. 8,9

Por lo tanto, la morfometría geométrica con sus avances nos puede ayudar a evidenciar el crecimiento y desarrollo del frenillo labial superior en cada una de las edades del infante, para así poder obtener información específica cuantificada de cómo y cuánto va creciendo esta estructura al pasar del tiempo, permitiéndonos reconocer lo fisiológico de la estructura o las desviaciones que puedan ocurrir en su trayecto, permitiendo así un diagnostico a tiempo y evidenciar el momento oportuno del tratamiento, sea logopedi$\mathrm{co}$, ortopédico o quirúrgico.

La presente investigación tiene como objetivo general realizar un análisis morfométrico del frenillo labial superior en diferentes etapas durante el crecimiento y desarrollo del niño en edades de 0 a 12 años de edad. Llegando a él a través de los siguientes objetivos específicos:

- Realizar un registro fotográfico digital del frenillo labial superior en niños de 0-12 años de edad del Municipio Naguanagua periodo Marzo-Septiembre 2016.

- Identificar los puntos anatómicos que comprenden el frenillo labial superior a través del software Makefan y tpsdig.

- Describir las variaciones morfometrícas del frenillo labial superior por cada grupo etario arrojadas por el software Morphoj, IMP7 y Past.

- Relacionar las variaciones morfométricas del frenillo labial superior entre todos los grupos etario a través del software Morphoj, IMP7 y Past.

\section{Materiales y Métodos:}

Para la presente investigación fundamentalmente se protegieron los derechos de los sujetos de estudio, primeramente bajo el consentimiento del representado y la Autorización en las Áreas de estudio dentro del Hospital Universitario "Ángel Larralde" así como el consentimiento institucional de la U.E. "Lago de Maracaibo", respetando los principios éticos de la investigación en seres humanos basado en la Declaración de Helsinki, siendo el proyecto de la presente investigación sometido a consideración por el comité de bioética del Hospital universitario "Ángel Larralde" bajo Aval en fecha 2 de Noviembre del 2016. 
La investigación fue de naturaleza Cuantitativa, siendo te Tipo Descriptivo con un diseño de Campo, teniendo como población los niños en edades comprendidas de 0 a 12 años de edad que acudieron al servicios de Materno-infantil y consulta control del niño sano, siendo estas Áreas pertenecientes al Hospital universitario “Ángel Larralde", y la U.E. "Lago de Maracaibo", ambas instituciones ubicadas en el Estado Carabobo - Venezuela. Se realizó un muestro No probabilístico de tipo intencional bajo los siguientes criterios:

Criterios de inclusión: Pacientes en edades comprendidas de 0 a 12 años, sanos, que estén recibiendo o hayan recibido lactancia materna mínimo hasta los 6 meses, con consentimiento informado debidamente por su representante.
Criterios de exclusión: Recién nacidos prematuros, Pacientes con alteración sistémica, que presenten alguna patología Síndromica, que hayan presentado traumatismos en el sector antero superior del maxilar, que utilicen o hayan utilizado alguna aparatología ortopédica u ortodoncia, con alteraciones a nivel maxilar, o con retardo en la erupción dental, con hábitos (Succión Digital, Deficiente respirador nasal).

La muestra obtenida fue de 260 niños en edades de 0 a 12 años, siendo clasificados en tres grupos: grupo de primera infancia que comprende niños en edades de 0-3 años, grupo de segunda infancia comprendiendo niños en edades de 36 años y tercera infancia correspondiente a niños en edades de 7-12 años, siendo distribuidos de la siguiente manera:

\begin{tabular}{|c|c|c|c|c|c|}
\hline \multicolumn{6}{|c|}{ Criterios Muéstrales } \\
\hline \multicolumn{2}{|c|}{ Muestra } & $\begin{array}{c}\text { Criterios de } \\
\text { muestra }\end{array}$ & $\begin{array}{l}\text { Cantidad } \\
\text { Estratificada }\end{array}$ & $\begin{array}{l}\text { Cantidad Total } \\
\text { de cada }\end{array}$ & $\begin{array}{c}\text { Resultado } \\
\text { Muestral }\end{array}$ \\
\hline \multirow{4}{*}{ HUAL } & \multirow{3}{*}{$\begin{array}{l}\text { Servicio de pediatría } \\
\text { Materno-infantil }\end{array}$} & Primera Infancia: & & \multirow{4}{*}{60} & \multirow{16}{*}{260} \\
\hline & & Recien nacido & 20 & & \\
\hline & & 1 año de edad & 20 & & \\
\hline & $\begin{array}{l}\text { Servicio Control } \\
\text { del niño sano }\end{array}$ & 2 años de edad & 20 & & \\
\hline \multirow{12}{*}{$\begin{array}{l}\text { E.B LAGO DE } \\
\text { MARACAIBO }\end{array}$} & $\begin{array}{l}\text { Preescolar } \\
\text { Nivel I }\end{array}$ & 3 años de edad & 20 & \multirow{6}{*}{80} & \\
\hline & \multirow{2}{*}{$\begin{array}{l}\text { Preescolar } \\
\text { Nivel II }\end{array}$} & Segunda Infancia: & & & \\
\hline & & 4 años de edad & 20 & & \\
\hline & $\begin{array}{c}\text { Preescolar } \\
\text { Nivel III }\end{array}$ & 5 años de edad & 20 & & \\
\hline & Primer Grado & 6 años de edad & 20 & & \\
\hline & Seounde Grodo & Tercera Infancia: & & & \\
\hline & Segundo Grado & 7 años de edad & 20 & \multirow{6}{*}{120} & \\
\hline & Tercer Grado & 8 años de edad & 20 & & \\
\hline & Cuarto Grado & 9 años de edad & 20 & & \\
\hline & Quinto Grado & 10 años de edad & 20 & & \\
\hline & \multirow{2}{*}{ Sexto Grado } & 11 años de edad & 20 & & \\
\hline & & 12 años de edad & 20 & & \\
\hline
\end{tabular}

Tabla 1. Distribución de la muestra según criterios muestrales. 
La recolección de la muestra se realizó a través de un registro fotográfico con una cámara digital NIKON® D3100 Réflex con un lente de estabilización de imagen VR NIKKOR® (18-55mm) equipada con Macro lens de $100 \mathrm{~mm}$ mas un ring flash genérico con luz Led, siendo mantenida en la misma posición con un trípode. La cámara se posicionó paralelamente al suelo tal que el eje focal de la cámara fuera paralelo al plano horizontal de referencia y centrado en medio del frenillo labial superior. Además, para evitar movimientos se solicitó ayuda de un asistente en la inmovilización de la cabeza del paciente. La distancia de la toma fue de $20 \mathrm{~cm}$ desde el lente a la cavidad bucal del paciente. (Imagen 1)

Para el procesamiento de imágenes y toma de puntos anatómicos se empleó el Programa Picasa, siendo posteriormente digitalizadas dichas imágenes bajo el software TpsUtil. Para la estandarización y disminución de error en la selección de puntos anatómicos se recurrió al complemento del Software IPM7, Makefan ; los Landmark se digi-

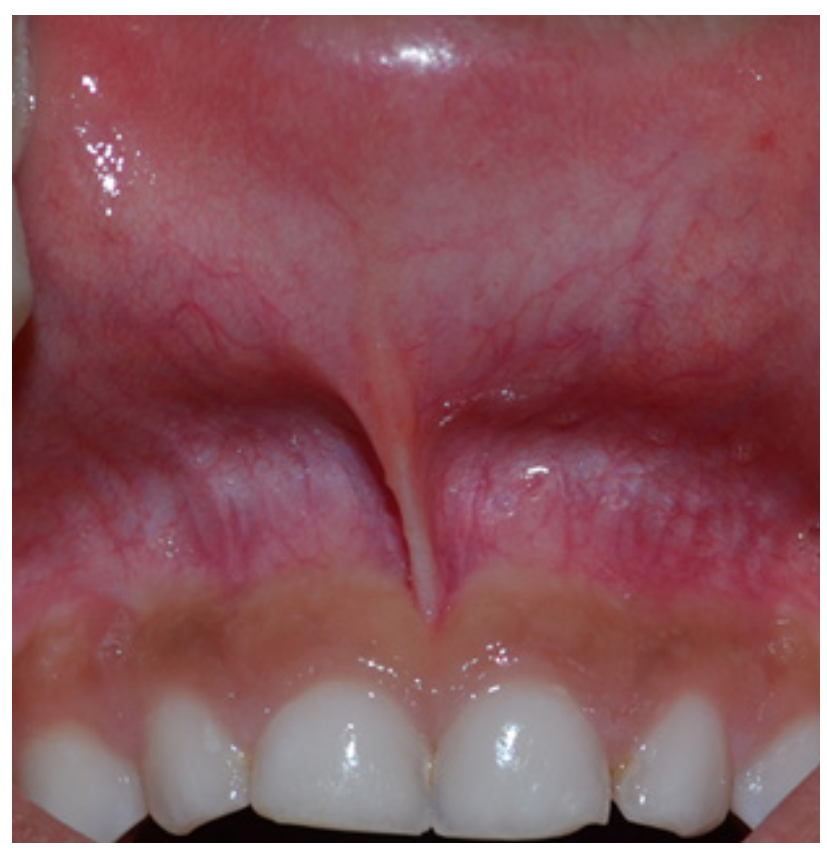

Imagen 1. Imagen obtenida como muestra de registro fotográfico.

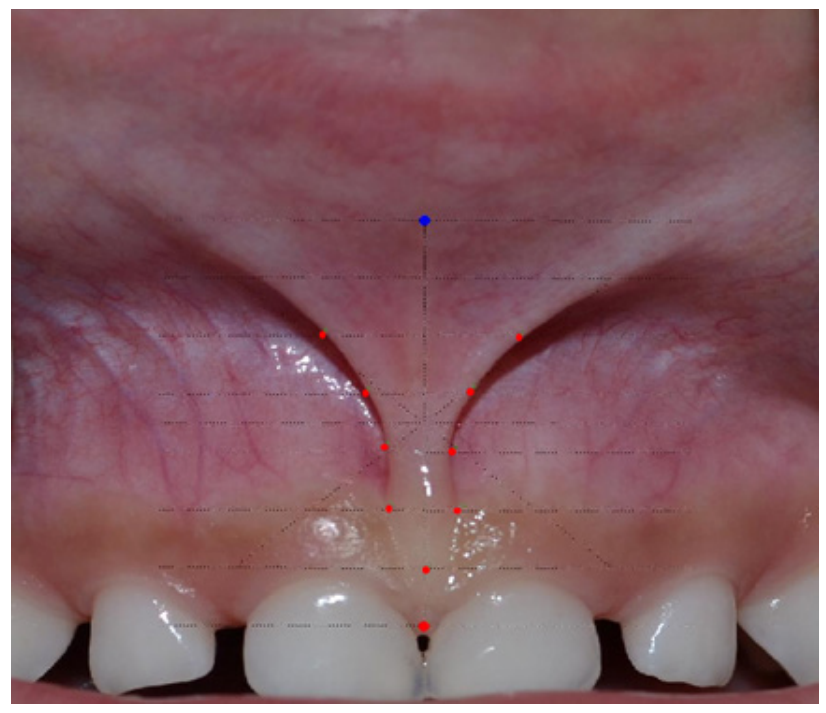

Imagen 2. Selección de "landmark" en Software TpsDig2.

talizaron dos veces en días diferentes, para reducir el error humano, se entraron los datos siempre en el mismo orden y el programa usado fue TpsDig v.2.16 que adquiere las coordenadas y captura los perfiles. (Imagen 2) 10,11,12. $^{1}$

En la presente investigación se hizo una selección de "landmark" para la identificación de puntos a localizar en la estructura anatómica de estudio. ${ }^{13,14}$.

Se emplearon puntos anatómicos( "landmark") de las tres categorías descritas por Bookstein (1991):15

- Tipo I: 4 "landmarks" bilaterales

- Tipo II: 4 "landmark" bilaterales

- Tipo III: 2 "landmark" bilaterales

Análisis Generalizado Procustes: Para el estudio de la forma se emplearon las coordenadas cartesianas de los Procrustes extrapoladas a través del proceso de superposición definido por Rohlf y Slice en 1990. Este proceso permite obtener 


\begin{tabular}{|c|c|c|}
\hline Landmark & Base anatómica & Tipo de Landmark \\
\hline 1 & Punto medio de la papila interdental de incisivos centrales superiores & Tipo I \\
\hline 2 & $\begin{array}{l}\text { Punto de inserción inferior del frenillo labial superior } \\
\text { en su porción inferior }\end{array}$ & Tipo I \\
\hline 3 & $\begin{array}{l}\text { Inicio de vertiente derecha del frenillo labial superior } \\
\text { en su porción inferior }\end{array}$ & Tipo II \\
\hline 4 & $\begin{array}{l}\text { Inicio de vertiente izquierda del frenillo labial superior } \\
\text { en su porción inferior }\end{array}$ & Tipo II \\
\hline 5 & $\begin{array}{l}\text { Porción media o Centro de la banda del frenillo labial } \\
\text { superior derecho }\end{array}$ & Tipo III \\
\hline 6 & $\begin{array}{c}\text { Porción media o Centro de la banda del frenillo labial } \\
\text { superior Izquierda }\end{array}$ & Tipo III \\
\hline 7 & $\begin{array}{l}\text { Inicio de vertiente derecha del frenillo labial superior } \\
\text { en su porción superior }\end{array}$ & Tipo II \\
\hline 8 & $\begin{array}{l}\text { Inicio de vertiente izquierda del frenillo labial superior } \\
\text { en su porción superior }\end{array}$ & Tipo II \\
\hline 9 & $\begin{array}{l}\text { Inicio de inserción del frenillo labial superior derecho } \\
\text { en su porción superior }\end{array}$ & Tipo I \\
\hline 10 & $\begin{array}{l}\text { Inicio de inserción del frenillo labial superior Izquierdo } \\
\text { en su porción superior }\end{array}$ & Tipo I \\
\hline
\end{tabular}

Autora: Cerrato 2016

Tabla 2. Identificación de "landmark" (puntos de referencia).

informaciones sobre la forma pura, eliminando las que se refieren a posición, orientación y dimensión rotando y trasladando las coordenadas de los "landmark"s (puntos de referencia) y refiriéndola a un Punto de consenso. Para el análisis Procustes se procesó el archivo tps preparado para cada grupo etario al programa IMP7. ${ }^{16,17}$.

Luego de la superposición, se analizaron los residuos de cada punto anatómico de referencia con respecto a la configuración de referencia para detectar y estudiar las diferencias

Deformación de placas delgadas: Se empleó el análisis de las deformaciones relativas, a partir de la inspección visual de las placas delgadas, el cual analiza la variación en la forma, descomponiendo la forma en sus componentes uniforme y no-uniforme. Las placas delgadas es una función suavizada que mapea todos los puntos en el espacio físico de la referencia (o consenso) sobre los puntos correspondientes en el espacio de los sujetos (Rohlf et al., 1996). El método consiste en ajustar la función de interpolación thin-plate spline a las coordenadas " $x, y$ " de los puntos de cada sujeto en una muestra. Para obtener los resultados de la deformación se procesaron los datos en el programa Past. Donde a traves de una gradiente de colores se visualiza la deformación correspondiente a cada sujeto de estudio. ${ }^{18}$

Deformaciones Relativas o Relative Warps: Se calcularon los relative warps los cuales son vectores de componentes principales en este espacio y son usados para describir las tendencias mayores en la variación en forma como deformaciones (variación no uniforme en forma) entre especímenes en una muestra. ${ }^{19}$ 
Líneas Equidistantes de Guía "Fan": Para la descripción del contorno del frenillo labial superior se crearon líneas equidistantes de guía ("Fan") a partir de la digitalización cada imagen de la muestra de estudio, logrando dividir la estructura de análisis con 8 líneas equidistantes y un abanico en el centro localizada en el centro de la misma. Esto con ayuda del programa MakeFan.

Análisis de Componentes Principales (ACP) y Análisis Canónico de Variables (ACV): . El ACP es una herramienta para simplificar descripciones de variación entre individuos, mientras que el ACV es usado para simplificar descripciones de diferencias entre grupos. Los análisis se realizaron con los programas y TpsRelw para el ACP, y CVAGen. ${ }^{16}$

Resultados: luego de la realización de las tomas fotográficas a la muestra seleccionada y tras haber sido estas imágenes procesadas y digitalizadas, se procedió al Análisis de las Variaciones Morfológicas del Frenillo labial Superior, siendo estudiadas bajo el concepto de morfometría geométrica Utilizando los siguientes métodos: 1 . Superposición, 2. Deformación, 3.Distancias Lineales y 4. Análisis

\section{X}

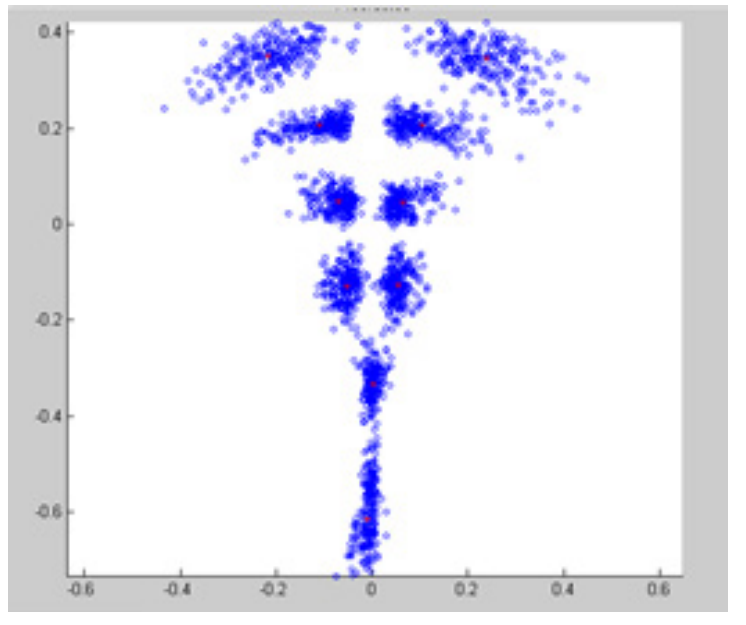

$Y$

Figura 1. Superposición Procrustes de puntos anatómicos del frenillo labial superior de una muestra de 260 niños. de Contornos. La muestra se clasifico en tres grupos primera infancia, segunda infancia y tercera infancia. Obteniendo que para los tres grupos hubo mayor superposición de puntos en la zona de inserción gingival correspondiente a los Landmark 1,2,3 y 10. Como se aprecia en la figura 1.

Siendo corroborado por el análisis de varianza, quien automáticamente organiza en orden de mayor a menor varianza los puntos anatómicos, el cual revela que la zona de inserción labial donde se presenta mayor variabilidad en todos los grupos etarios.

En relación al análisis de deformación, este se realizó en el programa Past el cual a través de una rejilla de deformación nos muestra donde la estructura se encuentra en desarrollo o crecimiento, siendo observado a través de una escala de colores, donde el color Azul refiere a cambios leves, siendo la zona de inserción papilar ubicada en esta coloración. El color Verde que refiere cambios moderados mostrando en esta coloración la zona media de la estructura de estudio y el rojo que refiera a la zona con mayor deformación cor-

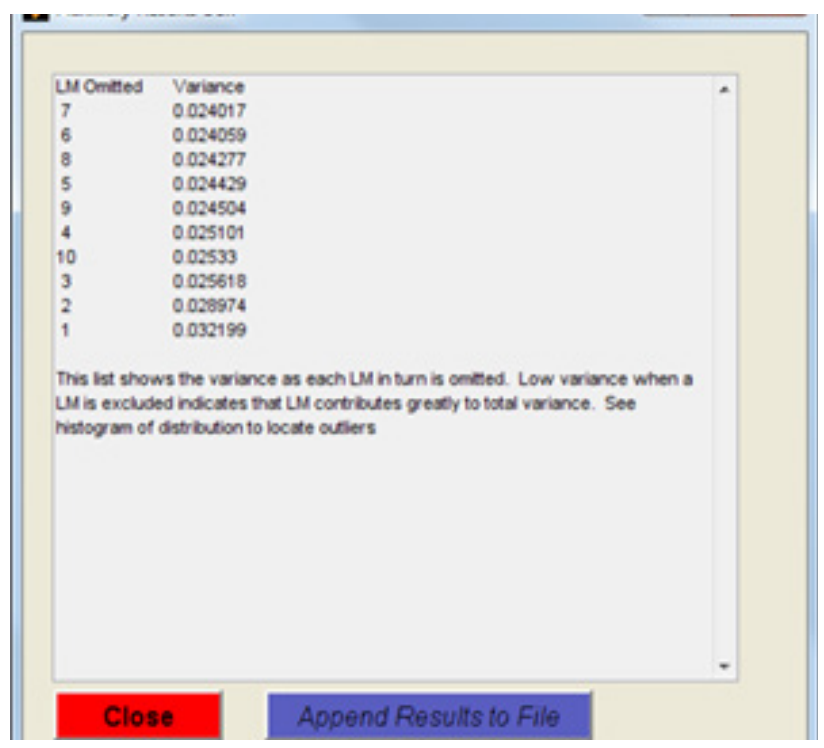

Figura 2. Tabla de varianza arrojada por el componente CoordGen del Software IMP7 para todas las edades. 

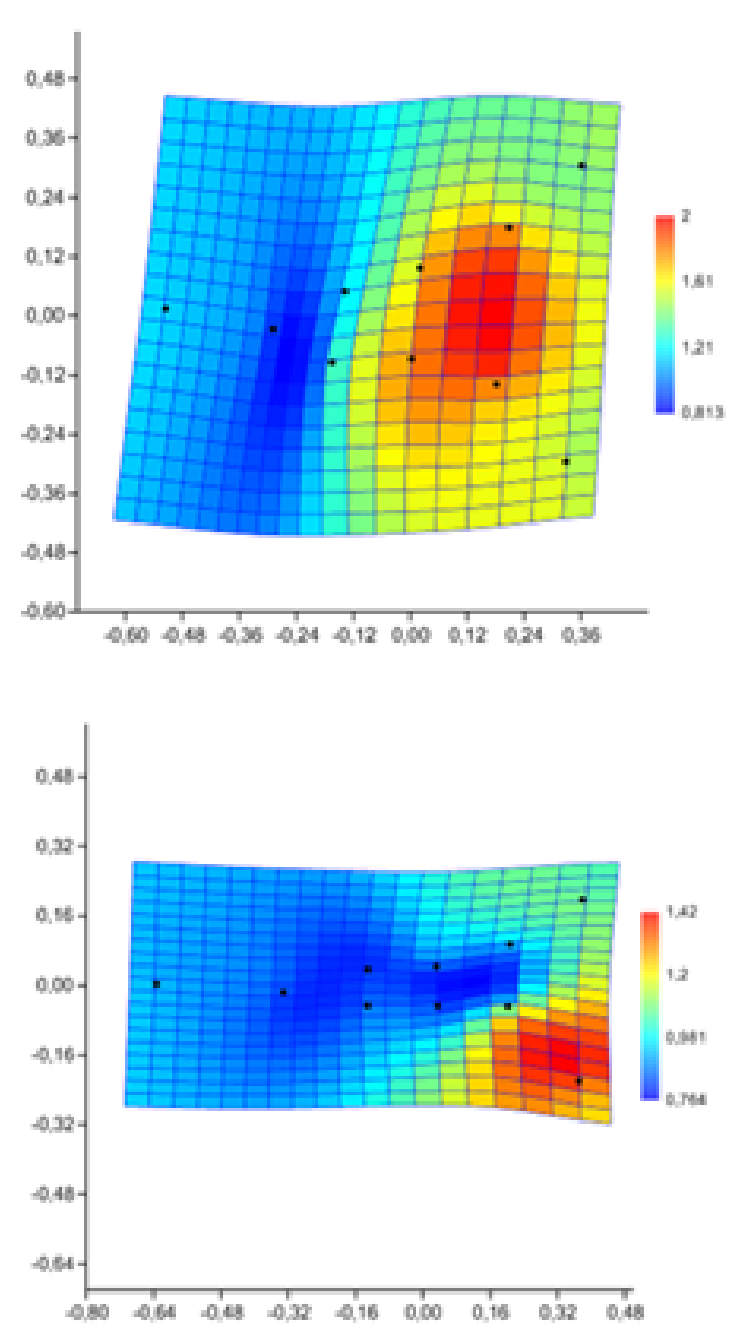
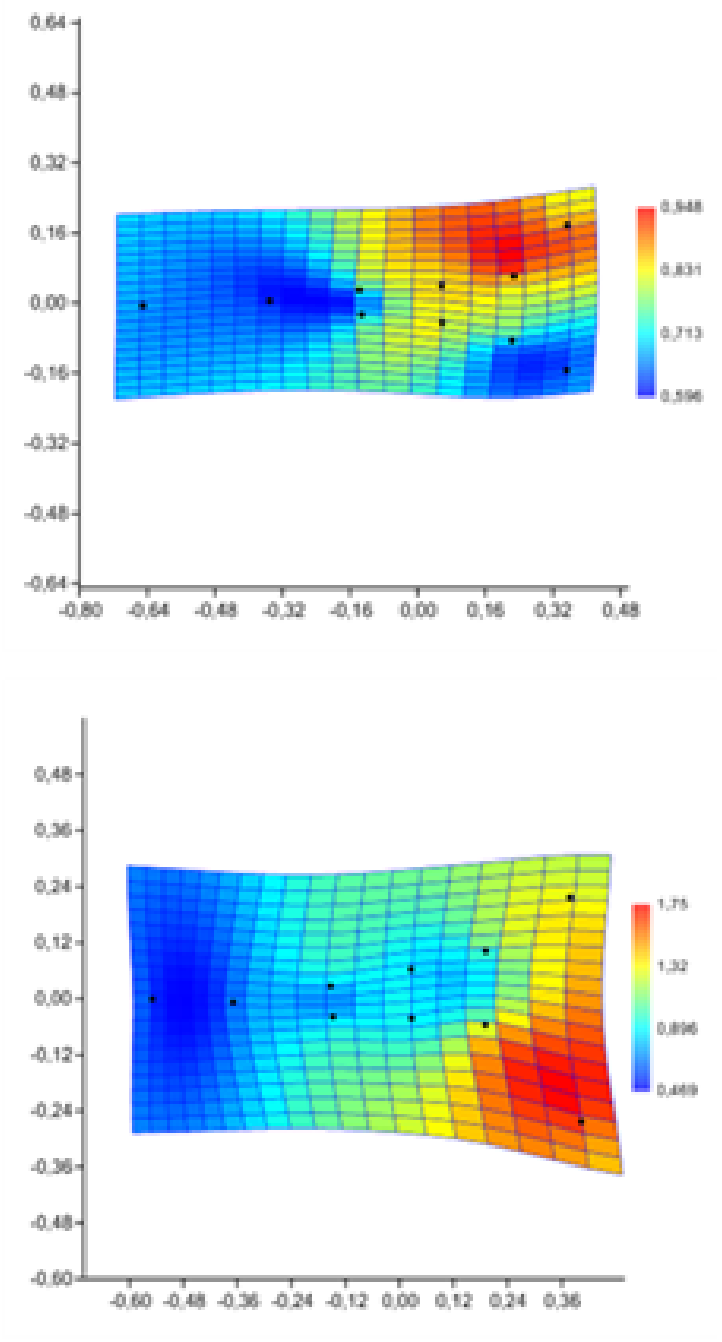

Figura 3w. Rejillas de deformación emitida por Past. Seleccionadas entre todas las edades.

respondiendo en este caso a la zona de inserción labial.

Ahora bien, con respecto al tamaño centroide o talla del frenillo labial superior, tenemos que en el análisis del frenillo labial superior en 260 individuos en edades comprendidas de R.N a 12 años se observa con el numero 2.0 el grupo R.N. el presento menor tamaño, mientras que en el resto de las edades no se observan diferencias claras.

Igualmente en la representación gráfica del ACP o Análisis de Componente Principal se pueden observar la clasificación de 260 individuos en 3 grandes correspondiendo con 1ra infancia para edades de R.N a 3 años, 2da Infancia correspondiente a edades de 3 años a 6 años y 3ra infancia correspondiente a edades de 7años a 12 años, resultando el grupo 1ra infancia correctamente reclasificado con un $71 \%$, el grupo de 2 da infancia correctamente reclasificado con un $57 \%$

Adicionalmente, el ACV o Análisis de Variables Canónicas se muestra que no hay separaciones congruentes de los componentes en estudio. Pudiéndose visualizar en las figuras resultantes de los análisis PCA y ACV a continuación reclasificado con un $66 \%$. 


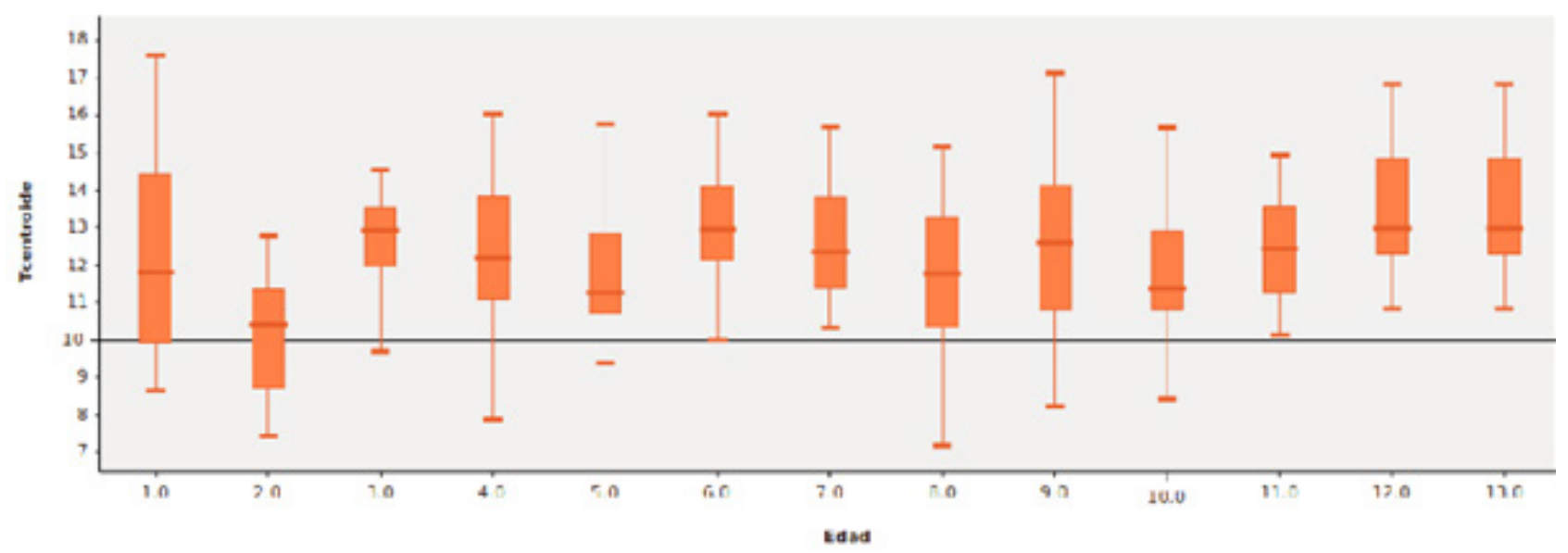

Gráfica 1. Representación gráfica del tamaño isométrico de 260 individuos en edades de R.N. a 12 años.

\section{Morfometría Tradicional}

Durante la investigación se realizaron una serie de medidas manuales del frenillo labial superior donde con vernier y un calibrador de metales se pudieron obtener siguientes resultados de promedio.

Estos resultados promedios presentaron leves variaciones con respecto al frenillo labial del recién nacido, y teniendo una desviación de $\pm 1 \mathrm{~mm}$

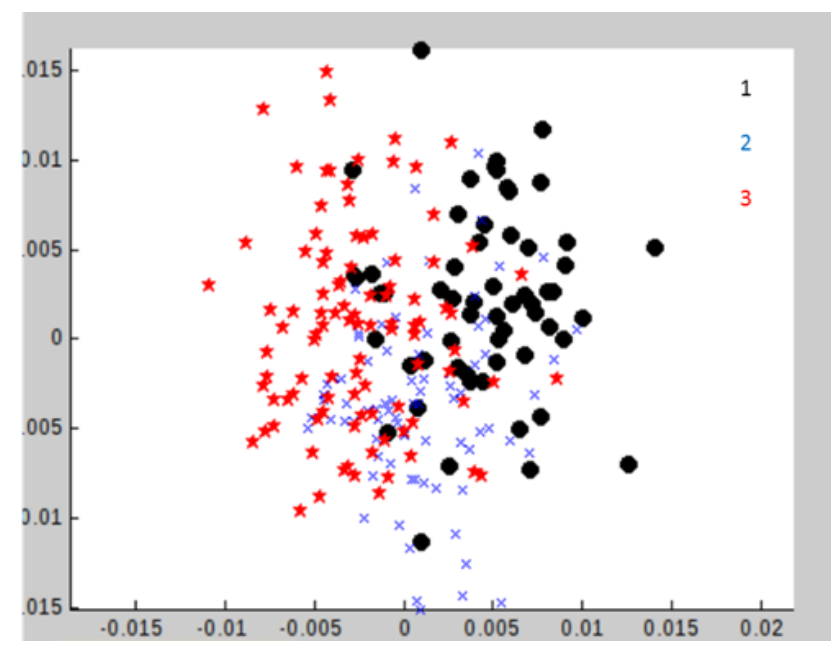

Figura 4. Resultado de PCA para muestra total de 260 individuos en grupos de 1ra infancia, 2da infancia y 3 ra infancia arrojado por el programa IPm7 bajo su componente PCAgen7. en todos los niños evaluados.

\section{DISCUSIÓN}

La morfometría geométrica es un campo realmente amplio para el estudio de diversas estructuras anatómicas y su dinámica, sin embargo en el campo de la ciencia de la salud así como la odontología su uso aún no ha sido totalmente aprovechado por lo que en Venezuela esta in-

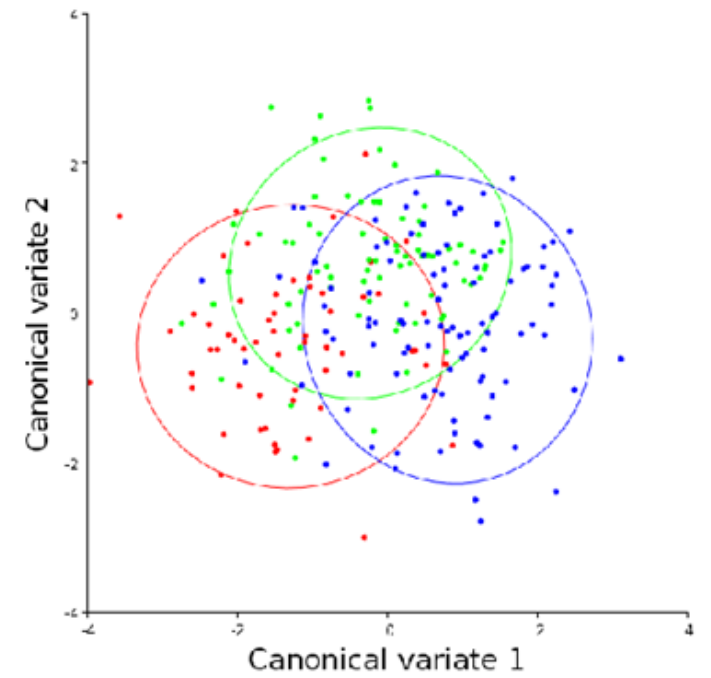

Figura 5. Resultado de ACV para muestra total de 260 individuos en grupos de 1ra infancia, 2da infancia y $3 r a$ infancia arrojado por el Programa IPm7 bajo su componente CVAgen 7 
vestigación viene siendo pionera sobre todo en el Área de Odontopediatría.

Por otra parte, con respecto al frenillo labial superior son diversas las controversias que rondan dicha estructura. En relación a las modificaciones que sufre el frenillo labial superior según el tipo de dentición. Nandanahousur y cols. En el año 2014 en su trabajo titulado "Morphology of maxillary labial frenum in primary, mixed, and permanent dentition of Indian children" concluyen que Se observó que el frenillo labial a medida que el niño avanzaba en años se observaba en condiciones más saludables. Mientras que el frenillo tectolabial persistente disminuyó proporcionalmente. ${ }^{7}$

Del otro modo, en la presente investigación se pudo obtener que el frenillo labial superior no presenta diferencias en las diferentes etapas del crecimiento y desarrollo, por lo que el tipo de dentición presente en cualquiera de las etapas de la infancia no influyen en la modificación del mismo. Igualmente el frenillo tectolabial debería disminuir de manera fisiológica antes de llegar al primer año de vida según lo analizado.

En relación a la inserción del frenillo labial superior, Sumita y Neeta de Nepal en el año 2012 estudiaron el "Frenillo labial superior en los niños de Nepal" teniendo como resultado wque tipo de frenillo labial más común fue el Gingival en $61.1 \%$ seguido del papilar penetrante en un $8,1 \%$ y teniendo que las diferencias entre los dos grupos etarios no fueron significativas. ${ }^{8}$

En tal sentido, durante el análisis de los resultados de la investigación se pudo observar que hay individuos que presentan leves modificaciones en cuanto a la inserción del frenillo a nivel gingival, teniendo una desviación estándar de 1 a $2 \mathrm{~mm}$, sin embargo en su inserción labial se observaron mayores modificaciones durante el crecimiento y desarrollo del niño pero en su mayoría con un desplazamiento horizontal.

Otro de los aspectos controvertidos con respecto al frenillo labial superior es su influencia en la formación del diastema interincisal. Álvarez 2013 realizo un estudio de tipo revisión bibliográfica con la finalidad de estudiar los factores etiológicos del diastema de la línea media superior, llegando a la conclusión de que la presencia del diastema puede ser atribuida a muchos factores: tipo de fusión de la sutura intermaxilar, disposición de las fibras transeptales del ligamento periodontal, frenillo labial patológico, dientes supernumerarios, ausencia congénita de incisivos laterales, quistes intermaxilares, discrepancias de tamaño dental, maloclusiones, hábitos, macroglosia y problemas sistémicos.

En este sentido, con base al estudio realizado, donde se obtuvo que el frenillo tectolabial característico del recién nacido debe evolucionar a su estado fisiológico durante el primer año de vida del niño. Podemos decir que la detección del frenillo patológico en conjunto con el cuadro clínico puede llevar al especialista a un tratamiento temprano interceptando el avance de diastemas inteincisales. Igualmente si se presentara una inserción del frenillo labial baja y sabiendo que su modificación durante el crecimiento y desarrollo será de 1 a $2 \mathrm{~mm} .{ }^{17}$

\section{CONCLUSIONES}

Luego de analizar los resultados obtenidos a través de los software de estudio se pudo determinar que en entre todos los grupos etarios la inserción interincisal no presenta cambios 
significativos, sino por lo contrario se mantiene sin modificaciones. En cuanto el crecimiento se pudo observar que la modificación que se puede presentar en el frenillo labial superior será en anchura en la zona de inserción labial.

En relación a la talla se puedo determinar que la misma no se modifica en los grupos de infantes a excepción de la edad R.N. la cual presenta una variación en talla siendo más pequeña que el resto natural por su condición tectolabial inicial. La medida promedio en el resto de las edades se estableció entre $11 \mathrm{~mm}$ a $13 \mathrm{~mm}$, variando entre 1-2mm de desviación en los distintos grupos.

Por otro lado, la inserción gingival entre los grupos etarios solo se obtuvo diferencia de $1 \mathrm{~mm}$ a $2 \mathrm{~mm}$ aproximadamente alejándose de la zona interincisal en la edad de R.N a 1 año de edad, denotándose que el frenillo labial del recién na- cido es tectolabial fisiológicamente y al 1er año de vida con dicha modificación entra en los rangos estándares de tamaño, inserción y forma que mantendrá a lo largo del desarrollo del niño, pudiendo así conocer que el desplazamiento del mismo en una inserción muy baja no mejorara una condición patológica presente.

Finalmente, es necesario saber que los resultados obtenidos nos llevan a replantearnos el enfoque del diagnóstico y el momento quirúrgico, por lo tanto podemos decir que la inserción baja del frenillo labial superior no presentara mejoras a lo largo de los años por lo que su planificación quirúrgica u ortopédica, ortodoncica, se pudiera hacer de manera profiláctica a temprana edad, siempre y cuando los signos clínicos y las herramientas diagnosticas indiquen su patología real.

\section{Referencias bibliográficas}

1. Esprella Vásquez José Antonio. Frenectomía. Rev. Act. Clin. Med Vol.2 Año 2012 [Revista En La Internet citado 8 de Junio de 2016 ].Disponible en: Http://Www.Revistasbolivianas.Org.Bo/Scielo.Php?Script=Sci_Arttext\&Pid=S230437682012001000003\&Lng=Es.

2. Díaz Me. Diastema Medio Interincisal Y Su Relación Con El Frenillo Labial Superior: Una Revisión. Rev Estomatol Herediana Vol. 14 Año 2004;14(1-2) :95 - 100[Revista En La Internet citado 8 de Junio de 2016 ]. http://www.upch.edu.pe/ vrinve/dugic/revistas/index.php/REH/article/view/2021

3. Dewel BF. The normal and the abnormal labial frenum clinical differentiation. J Am Dent Assoc 1946; 33(3):318-329.

4. Toro Ibacache María Viviana, Manriquez Soto Germán, Suazo Galdames Iván. Morfometría Geométrica Y El Estudio De Las Formas Biológicas: De La Morfología Descriptiva A La Morfología Cuantitativa.Int.J.Morpho.2010 [Revista En La Internet citado 12 de Junio de 2016].Disponible en: Http://Www.Scielo.Cl/Scielo.Php?Script=Sci_Arttext\&Pid=S0717

5. Nicolás Jaramillo-O. Morfometría Geométrica: Principios Teóricos Y Métodos De Empleo.Año 2011. [Revista En La Internet citado 15 de Julio de 2016] Disponible En:http://Www.Researchgate.Net/Publication/237522938_Morfometria_Geometrica_

Principios_Teoricos_Y_Metodos_De_Empleo

6. Townsend JA, Brannon RB, Cheramie T, Hagan J. Prevalence and variations of the median maxillary labial frenum in children, adolescents, and adults in a diverse population. Gen Dent. 2013 vol 2 , 57-60.

7. Nandanahour, Nagaveni NB, Umashankara KV. Morphology of maxillary labial frenum in primary, mixed, and permanent dentition of Indian children. J Cranio Max Dis 2014. 3, 5-10 
8. SumitaUpadhyay, Neeta Ghimire. Attachment of maxilary labial frenum in nepalese children. Año 2012. Ortho journal of Nepal. Vol. $2 \mathrm{~N}^{\circ} 1$,

9. Kacar, D.; Cakmak, F.; Dogan, A.; Sevinc, O. \& Barut, C. Evaluation of lingual frenulum using geometric morphometrics. Año 2011. Int. J. Morphol., 29(2):313-317

10. Toro, I. M. V.; Manriquez, S. G. \& Suazo, G. I. Morfometría geométrica y el estudio de las formas biológicas: de la morfología descriptiva a la morfología cuantitativa. Int. J. Morphol., Año 2010. 28(4):977-990.

11. Adams, D. C.; Rohlf, F. J. \& Slice, D. E. Geometric morphometrics: ten years of progress following the "revolution". Ital. J. Zool.,Año 2004. 71:5-16.

12. Bookstein, F. L. Morphometric tools for "landmark" data:Geometry and biology. Cambridge, Cambridge University Press, Año 1991.

13. Bookstein, F. L. Landmark methods for forms without "landmark"s: Morphometrics of group differences in outline shape. Med. Image Anal., Año 1997.1(3):225-43

14. Marcus, L. F. Traditional morphometrics. En: Proceedings of the Michigan Morphometrics Workshop. Rohlf, F. J. \& Bookstein, F. L. (Eds). Special Publication Number 2. Ann Arbor, Michigan, The University of Michigan Museum of Zoology, año 1990. pp.77-122.

15. Rohlf, F. J. Shape statistics: Procrustes superimpositions and tangent spaces. J. Classification, Año 1990. 16:197-223. 16. Slice, D. E. Geometric Morphometrics. Año 2007. Ann. R. Anthr.,36:261-8.

17. Alvarez, Tatiana. McQuattie, Isabel. Scannone, Ana Cristina. Factores Etiológicos del Diastema de la línea media superior: Revisión de la literatura Revista Latinoamericana de Ortodoncia y Odontopediatría Vol.15. Año 2013 [Revista En La Internet citado 17 de Julio de 2016] FUENTE: http://www.ortodoncia.ws/publicaciones/2013/art36.asp

Recibido: 30/12/2016

Aceptado: 21/02/2017

Correspondencia: Osmary Darisay Cerrato López, cerratoosmary@gmail.com Av. José Antonio Páez, Conjunto Residencial “El Rosal” Torre C, apto. 14-C sector La Candelaria. Tinaquillo Estado Cojedes. Venezuela. Teléfono: +5802587664465 o 